\title{
ON MEAN VALUE LIMITS \\ FOR BOUNDED REGULAR FUNCTIONS
}

\author{
by N. A. BOWEN \\ (Received 24th December 1963)
}

\section{Introduction}

The object of this note is to state and prove two theorems of the nature of Montel's Limit Theorem for a function which is regular and bounded in a region $G$, but involving as hypothesis the limit of a mean value of the function instead of the limit of the function itself. Theorem 2 below (with $b=b^{\prime}=1$ ), in which $G$ is a half-strip, was stated several years ago in a letter to A. J. Macintyre and myself from J. M. Whittaker, who added that it could be proved by integrating the inequality in Lemma 3 below (which is due to Dr Whittaker). $\dagger$ As far as I can discover, such a proof still has not appeared in print; hence the present one.

In Theorem 1 below, in which $G$ is a half-plane, the mean value hypothesis is more effective, and, by a different method, I obtain uniformly over any interior sector the limit of the function itself, as in Montel's Theorem.

Theorem 1. Let $f(z)$ be regular and $|f(z)|<M$ in the right half-plane $\mathscr{R}(z)>0$ and let

$$
T^{-1} \int_{0}^{T}\left|f\left(r e^{i \alpha}\right)-l\right|^{b} d r \rightarrow 0 \text { as } T \rightarrow \infty
$$

\}

where $l, b(>0), \alpha$ are constants and $-\frac{1}{2} \pi<\alpha<\frac{1}{2} \pi$.

Then

$$
f(z) \rightarrow l \text { as }|z| \rightarrow \infty
$$

uniformly in $|\arg z| \leqq \frac{1}{2} \pi-\delta$ for every $\delta$ in $0<\delta<\frac{1}{2} \pi$.

$$
\}
$$

Proof of Theorem 1. Let

$$
F(z) \equiv f(z)-l
$$

We shall need the following

Lemma 1. If $\phi(r) \geqq 0$ is measurable and

$$
T^{-1} \int_{0}^{T} \phi(r) d r \rightarrow 0 \text { as } T \rightarrow \infty,
$$

† My thanks are due to Dr Whittaker for bringing this and other problems to my notice, and for his kind permission to make use of them. 
then

$$
\phi(r) \rightarrow 0
$$

as $r \rightarrow \infty$ through a set of linear density unity.

The proof of this lemma, being straightforward, is omitted.

Theorem 1 follows immediately from (1), (3), Lemma 1 and

Lemma 2. $\dagger$ Let $g(z)$ be regular and bounded in $\mathscr{R}(z)>0$, let inf $\left|g\left(r e^{i \theta}\right)\right|$, $\left(|\theta|<\frac{1}{2} \pi\right)$ tend to zero in a set $E$ having the property $\lim _{r \rightarrow \infty} r^{-1} m(r)>0$, where $m(r)$ denotes the measure of the part of $E$ contained in the interval $(0, r)$. Then, as $z \rightarrow \infty, g(z) \rightarrow 0$ uniformly in $|\arg z| \leqq \frac{1}{2} \pi-\delta,(\delta>0)$.

Theorem 2. Let $f(z)$ be regular and $|f(z)|<M$ in the half-strip $|\mathscr{R}(z)|<1$, $\mathscr{F}(z)>0$ and let

$$
T^{-1} \int_{0}^{T}|f(a+i y)-l|^{b} d y \rightarrow 0 \text { as } T \rightarrow \infty
$$

where $l, b, a$ are constants, $b$ is positive and $-1<a<1$.

Then

$$
\left.T^{-1} \int_{0}^{T}|f(x+i y)-l|^{b^{\prime}} d y \rightarrow 0 \text { as } T \rightarrow \infty\right)
$$

uniformly in $|\mathscr{R}(z)| \leqq 1-\delta$ for every $\delta$ in $0<\delta<1$, where $b^{\prime}$ is an arbitrary positive constant.

Proof of Theorem 2. The natural approach here would be to apply the transformation

$$
-\frac{1}{2} i \pi z=\log Z
$$

in the hope of obtaining the conditions of Theorem 1 , with $Z$ in place of $z$. It is easily seen that this fails, since the analogue of (1) would have an additional factor $r^{-1}$ (using the original notation) in the integrand and it would not be possible to deduce the existence of a linear set $E$ with the property required for Lemma 2. However, as stated in the Introduction, Theorem 2 may be proved directly, with the aid of

Lemma 3.+ Let $h(z)$ be regular and $|h(z)| \leqq M^{\prime}$ in $|z|<1$, and let

$$
\left|h\left(a_{k}\right)\right| \leqq L\left(k=0,1,2, \ldots, N^{\prime}\right),
$$

the $a_{k}$ being any distinct points in $|z| \leqq \omega<1$. Then

$$
\begin{gathered}
|h(z)| \leqq M^{\prime}\left(\frac{2 \omega}{1+\omega^{2}}\right)^{N^{\prime}+1}+\frac{6 L \Delta(2 \omega)^{N^{\prime}}}{\left(1-\omega^{4}\right)}, \quad(|z| \leqq \omega) \\
+ \text { See (1). } \\
\ddagger \text { (2, p. 57). }
\end{gathered}
$$


where

$$
\Delta=\sum_{k=0}^{N^{\prime}} \prod_{j}^{\prime}\left|a_{j}-a_{k}\right|^{-1},
$$

the dash attached to $\Pi$ implying that the factor for which $j=k$ is to be omitted.

In order to prove Theorem 2, we first set

$$
F(z) \equiv f(z)-l
$$

and apply Lemma 1 as before.

We find, using (4), that, for arbitrary $\varepsilon>0$,

$$
|F(z)|<\varepsilon, \quad\{\mathscr{R}(z)=a\}
$$

on a set of points whose measure $m(T)$ satisfies

$$
\begin{aligned}
& \qquad T^{-1} m(T)>1-\varepsilon \\
& \text { for all } T>T_{0}(\varepsilon)
\end{aligned}
$$

As the rest of the proof of Theorem 2 consists of a number of steps, it may be worthwhile to state the argument briefly before giving the details. From (7) we show that there are circles, centred on the imaginary axis and of radii $\left(1-\frac{1}{2} \delta\right)$, each containing as many distinct points, not too close together, at which (6) holds, as we please. Hence, Whittaker's lemma gives $|F(z)|<\varepsilon^{\prime}$ $\left(\varepsilon^{\prime}>0\right.$ arbitrarily small when $\varepsilon$ is chosen small) throughout such circles, and, as these circles are shown to cover most of the strip

$$
|\mathscr{R}(z)| \leqq 1-\delta, \quad 1<\mathscr{I}(z)<T,
$$

the required result follows on summation of appropriate integrals taken along chords of the circles, the contribution from the remaining parts of the strip being insignificant, since $|F(z)|$ is bounded.

Remaining details of the proof of Theorem 2. Given any $T>0$, let $\Gamma_{\mu}(\mu$ $=1,2,3, \ldots, n)$ denote the sequence of equal circles

$$
\left|z-i y_{\mu}\right|=1-\frac{1}{2} \delta \quad(\delta>0 \text { arbitrary })
$$

where $y_{1}=1, y_{\mu}-y_{\mu-1}=\lambda(\delta)$ for $\mu=2,3, \ldots, n$, where $\lambda(\delta)=\lambda$, say, is the length of the chord $C_{\mu}$ cut off the line $\mathscr{R}(z)=1-\delta$ by the circle $\Gamma_{\mu}$, and $n$ is chosen so that

$$
n \lambda \geqq T>(n-1) \lambda \text {. }
$$

Then each point of the strip $|\mathscr{R}(z)| \leqq 1-\delta, 1 \leqq \mathscr{I}(z)<T$ lies inside or on at least one of the sequence of adjoining rectangles $R_{\mu}(\mu=1,2,3, \ldots, n)$ which have their centres on the imaginary axis and a chord $C_{\mu}$ for a side.

Let $c_{\mu}(a)$ with the $a$ of (4) denote the segment cut off the line $\mathscr{R}(z)=a$ by the rectangle $R_{\mu}$, and let us call the corresponding circle $\Gamma_{\mu}$ " satisfactory" if (6) holds in $c_{\mu}(a)$ over a length not less than $\lambda\left(1-2 \varepsilon^{\frac{1}{2}}\right)$.

$$
\text { E.M.S. }-\mathrm{H}
$$


Lemma A. For $T>T_{0}^{\prime}(\varepsilon)$ the number $p$ of " satisfactory" circles $\Gamma_{\mu}$ is at least $\dagger\left[n\left(1-\varepsilon^{\frac{1}{2}}\right)\right]$.

Proof of Lemma A. By (7) we know that the measure of the intervals over which (6) holds exceeds $T(1-\varepsilon)$ and since, with $T>T_{0}^{\prime}(\varepsilon) \geqq T_{0}(\varepsilon)$, this by (8) includes a length of at least $n \lambda(1-2 \varepsilon)$ measured along the segments $c_{\mu}(a)$ $(\mu=1,2,3, \ldots, n)$, then, for at least one of the circles $\Gamma_{\mu},(6)$ holds on the corresponding $c_{\mu}(a)$ over a length not less than $\lambda(1-2 \varepsilon)$. Removing one such circle, we have a total length at which (6) holds of at least $\lambda\{h(1-2 \varepsilon)-1\}$ measured along the segments $c_{\mu}(a)$ of the remaining $(n-1)$ circles, on at least one $c_{\mu}(a)$ of which (6) therefore holds over a length of at least $\lambda\left\{1-\frac{2 \varepsilon n}{n-1}\right\}$. Proceeding in this way we find that, for the $p$ th such circle, (6) holds over a length $\lambda^{\prime} \geqq \lambda\left\{1-\frac{2 \varepsilon n}{n-p+1}\right\}$ of the corresponding segment $c_{\mu}(a)$. If we set $p=\left[n\left(1-\varepsilon^{\frac{1}{2}}\right)\right]$, we have $\lambda^{\prime}>\lambda\left(1-2 \varepsilon^{\frac{1}{2}}\right)$, and Lemma A follows.

Lemma B. Given $\varepsilon^{\prime}>0$, if $\varepsilon>0$ is chosen sufficiently small, then $|F(z)|<\varepsilon^{\prime}$ throughout every "satisfactory" circle $\Gamma_{\mu}$.

Proof of Lemma B. In any " satisfactory" circle $\Gamma_{\mu}$ let us divide the rectangle $R_{\mu}$ into $N$ equal strips $\ddagger$ of width $N^{-1} \lambda$ by drawing the appropriate horizontal lines, $N$ being a large positive integer to be specified later. It may happen that (6) fails to hold at every point of the corresponding $c_{\mu}(a)$ inside some of these strips. If so, let $N_{1}$ denote the number of such strips. Since $\Gamma_{\mu}$ is "satisfactory", the total width $N^{-1} N_{1} \lambda$ of these strips cannot exceed $2 \varepsilon^{\frac{1}{2}} \lambda$, whence $N_{1} \leqq 2 \varepsilon^{\frac{1}{2}} N$. Thus there are points in each of at least $v=N-\left[2 \varepsilon^{\frac{1}{2}} N\right]$ of these strips, at which (6) holds, and, working in the direction of increasing $y$, we select one such point in the first strip, one in the third, and so on, taking alternate strips only. We thus find $v^{\prime}+1\left(=\frac{1}{2} v\right.$ or $\frac{1}{2}(v+1)$ according as $v$ is even or odd) distinct points in $\Gamma_{\mu}$ at which (6) holds and for the $\Delta$ of Lemma 3 we have the inequality

$$
\Delta \leqq \frac{1}{\left(N^{-1} \lambda\right)^{v^{\prime}}} \sum_{u=0}^{v^{\prime}} \frac{1}{u !\left(v^{\prime}-u\right) !}=\frac{2^{v^{\prime}}}{\left(N^{-1} \lambda\right)^{v^{\prime}}\left(v^{\prime}\right) !}<\left\{2 e / N^{-1} \lambda v^{\prime}\right\}^{v^{\prime}},
$$

which, taking the circle $\Gamma_{\mu}$ in question as the interior circle $|z| \leqq \omega$ of the lemma, and $F(z)$ in place of $h(z)$, gives $\S$ by (6) the inequality

$$
\begin{array}{r}
6 L \Delta(2 \omega)^{N^{\prime}} /\left(1-\omega^{4}\right)<12 \delta^{-1} \varepsilon\left\{2 e(2-\delta) / N^{-1} \lambda v^{\prime}\right\}^{v^{\prime}}<12 \delta^{-1} \varepsilon\left(16 \lambda^{-1} e\right)^{N} \\
=\varepsilon K(N, \delta), \text { say, }
\end{array}
$$

$\dagger$ As usual $\left[n\left(1-\epsilon^{\frac{1}{2}}\right)\right]$ denotes the integral part of $n\left(1-\epsilon^{\frac{1}{2}}\right)$.

\$ Compare (3), p. 546 .

$\S$ Remembering the definition of a " satisfactory" circle $\Gamma_{\mu}$. 
for $N>8$, if we assume (as we obviously may) that $0<\varepsilon<2^{-6}$. The circle $|z|<1$ of Lemma 3 is here the unit circle concentric with $\Gamma_{\mu}$, of course, and we get

$$
\left.\begin{array}{rl}
M^{\prime}\left(\frac{2 \omega}{1+\omega^{2}}\right)^{N^{\prime}+1} & =M\left\{\frac{2-\delta}{1+\left(1-\frac{1}{2} \delta\right)^{2}}\right\}^{v^{\prime}+1} \\
& =M \kappa^{v^{\prime}+1}, \text { say }, \\
& <M \kappa_{1}^{N}
\end{array}\right\}
$$

where, for arbitrary $\delta$ in $0<\delta<1, \kappa_{1}=\kappa_{1}(\delta)$ satisfies the inequalities

$$
0<\kappa<\kappa_{1}<1 \text {. }
$$

By (9), (10) and (11), Lemma 3 applied to $F(z)$ thus gives

$$
\begin{aligned}
|F(z)| & <M \kappa_{1}^{N}+\varepsilon K(N, \delta) \quad\left(|z| \leqq 1-\frac{1}{2} \delta\right) \\
& <\frac{1}{2} \varepsilon^{\prime}+\frac{1}{2} \varepsilon^{\prime} \quad\left(\varepsilon^{\prime}>0 \text { arbitrary }\right) \\
& =\varepsilon^{\prime}
\end{aligned}
$$

by first choosing $N$ sufficiently large and then $\varepsilon$ sufficiently small. This proves Lemma $B$.

We are now in a position to complete the proof of Theorem 2. The inequality (12) holds over every " satisfactory" circle and a fortiori over every satisfactory rectangle $R_{\mu}$. Integrating along the lines $\mathscr{R}(z)=$ constant and remembering that, by (3), $|F(z)|<M+|l|=M_{1}$, say, we thus have uniformly for $|\mathscr{R}(z)| \leqq 1-\delta$

$$
\begin{aligned}
& T^{-1} \int_{0}^{T}|F(x+i y)| d y \leqq T^{-1}\left\{\int_{0}^{T_{0}^{\prime}}+\int \text { satisfactory " } c_{\mu}(x)\right. \\
&\left.+\int \text { “ unsatisfactory " } c_{\mu}(x)\right\} \\
&<T^{-1}\left\{M_{1} T_{0}^{\prime}+\varepsilon \lambda n+M_{1} \lambda\left(\varepsilon^{\frac{1}{2}} n+1\right)\right\}
\end{aligned}
$$

by (12) and Lemma $A$,

$$
<T^{-1} M_{1}\left(T_{0}^{\prime}+\lambda\right)+\varepsilon^{\prime}+M_{1}\left(\varepsilon^{\prime}\right)^{\frac{1}{2}}
$$

by (8),

$$
<T^{-1} M_{1}\left(T_{0}^{\prime}+2\right)+\varepsilon^{\prime}+M_{1}\left(\varepsilon^{\prime}\right)^{\frac{1}{2}} .
$$

Hence

$$
T^{-1} \int_{0}^{T}|F(x+i y)| d y<\varepsilon^{\prime \prime} \quad\left(\varepsilon^{\prime \prime}>0 \text { arbitrary, } T>T_{0}^{\prime \prime}\left(\varepsilon^{\prime \prime}\right)\right)
$$

uniformly for $|\mathscr{R}(z)| \leqq 1-\delta$, since the second and third terms on the right hand side of (13) are each less than $\frac{1}{3} \varepsilon^{\prime \prime}$ provided that $\varepsilon$ is taken sufficiently small, and with the corresponding $T_{0}^{\prime}(\varepsilon)$ (of Lemma A) the first term is less than $\frac{1}{3} \varepsilon^{\prime \prime}$ for all $T>T_{0}^{\prime \prime}\left(\varepsilon^{\prime \prime}\right) \geqq T_{0}^{\prime}(\varepsilon)$. 
Theorem 2 for the case $b^{\prime}=1$ follows from (3) and (14). Clearly only minor modifications are needed in the last step of the proof, viz., the " integration " step, in order to obtain Theorem 2 with arbitrary $b^{\prime}>0$.

In conclusion it may be worth-while to note that in the hypotheses of both Theorems 1 and 2 the straight line of integration may be replaced by any curve $\Gamma$ interior to the region of definition along which $|z|$ tends steadily to infinity with sufficient rapidity. We could for example ask that $\lim _{r \rightarrow \infty} \frac{d r}{d s} \geqq \eta>0$ along $\Gamma, r, s$ denoting the usual polar coordinate and the length of arc respectively.

My thanks are due to Dr R. O. Davies, who kindly read the original version of this paper and offered numerous suggestions for clarifying the arguments involved.

\section{REFERENCES}

(1) T. HaLl, Arkiv för Mat., Ast. och Fysik, 25A, No. 28, (1937), 1-8.

(2) J. M. WhITTAKeR, Interpolatory Function Theory (Cambridge, 1935).

(3) J. M. Whittaker, Proc. London Math. Soc., (2) 41 (1936), 544-552.

\section{THE UNIVERSITY}

LEICESTER 